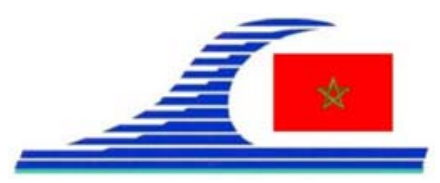

Conférence Méditerranéenne Côtière et Maritime

EDITION 2, TANGER, MAROC (2011)

Coastal and Maritime Mediterranean Conference

Disponible en ligne - http://www.paralia.fr - Available online

\title{
Stratégies pour une communication durable entre l'étang d'Urbino et la mer
}

\author{
Julie LEBUNETEL ${ }^{1}$, Pierre FARNOLE ${ }^{1}$
}

\author{
1. ERAMM, 1503 rte des Dolines, BP 42, 06901 Sophia Antipolis, France. \\ jl@eramm.fr ; pf@eramm.fr
}

\section{Résumé :}

L'étang d’Urbino est un espace naturel préservé exceptionnel de la plaine orientale corse. Les activités professionnelles présentes sur site sont réalisées avec respect envers le milieu naturel et de manière à conserver la ressource halieutique du site. La problématique réside dans le manque de communication entre l'étang et la mer qui met en péril la faune marine. Le grau, ouverture dans le lido, a subi des changements conséquents au fil des ans induisant une augmentation du linéaire de chenal. Cela génère des difficultés d'entretien et une communication non pérenne entre la mer et l'étang. Une analyse hydrosédimentaire à partir d'une étude conjointe de terrain et de modélisation numérique a été réalisée. Les mécanismes locaux responsables de la sédimentation dans le chenal à l'origine du manque de communication entre l'étang et la mer ont pu être déterminés. Dans l'objectif d'aboutir à une solution permettant de réactiver durablement les échanges, plusieurs actions ont été proposées visant notamment à réduire de manière significative les apports sédimentaires dans le chenal.

Mots-clés :

Aménagement côtier - Dynamique littorale - Modélisation numérique Hydrodynamique - Transport sédimentaire

\section{Introduction}

\subsection{Description du site d'étude}

L'étang d’Urbino, d'une superficie de 790 ha, se situe sur la commune de Ghisonaccia, sur la façade Est de la Corse dans le département de Haute-Corse. L'étang d'Urbino est la propriété du Conservatoire du Littoral. Avec une profondeur maximale de $9 \mathrm{~m}$, c'est le deuxième étang le plus profond de Corse. Les apports en eau douce du bassin versant sont faibles (MONTIGNY, 2008). Les échanges avec la mer s'effectuent par l’intermédiaire d’un grau situé au nord du lido.

\subsection{Contexte hydrosédimentaire de la façade maritime}

Le transit sédimentaire littoral est globalement dirigé vers le Nord. Une étude récente sur l'évolution du trait de côte le long de la plaine orientale (BRGM, 2010) a montré que celui-ci était en recul de 0 à $20 \mathrm{~m}$ sur ce secteur excepté à proximité immédiate du 
La connaissance de la Mer :

un vecteur du développement durable en Méditerranée

grau. Le grau artificiel crée une discontinuité du trait de côte. Il joue un rôle d'épi, créant ainsi une accrétion au sud de l'ouverture et une érosion au nord (voir figure 1a).

\subsection{Historique des échanges entre l'étang et la mer}

Avant les années 70, les échanges hydrauliques entre la mer et l'étang s'effectuaient par le biais d'un grau naturel, au sud du lido sableux. Puis, le développement des activités piscicoles a induit le besoin d'augmenter les échanges entre l'étang et la mer. Ainsi le grau artificiel fut mis en place sur la partie nord du lido, facile d'accès. Constitué de palplanches en acier, il mesurait une centaine de mètres de longueur. Au cours des années, et via notamment l'entretien du chenal, des zones de dépôt ont été réalisées au sud et au nord du lido côté étang (BRGM, 1996). Ensuite, l'accumulation de sédiments au droit du chenal côté étang fut contournée via la mise en place d'une langue de sédiments. Ainsi le chenal mesure maintenant 350 m de longueur (voir figure 1a).

L'entretien d'un tel linéaire de chenal engendre une logistique considérable (en termes de moyens techniques et financiers). A l'heure actuelle, la profondeur d'eau est faible $(50 \mathrm{~cm})$ et les périodes où le chenal est obstrué par une barre sableuse ne sont pas rares (voir figure 1b). Le manque d'oxygénation du plan d'eau induit par le manque d'échanges avec la mer n'est pas favorable car cela met en péril la faune marine et compromet le recrutement du poisson.

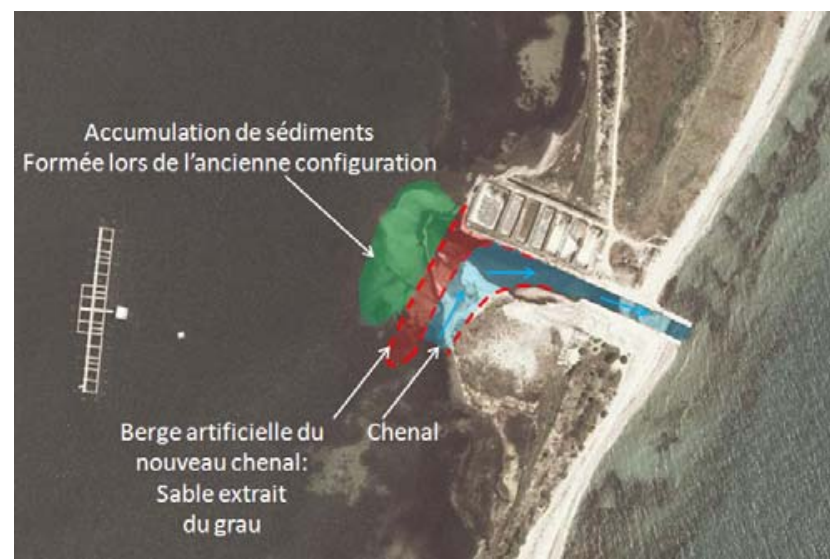

(a)

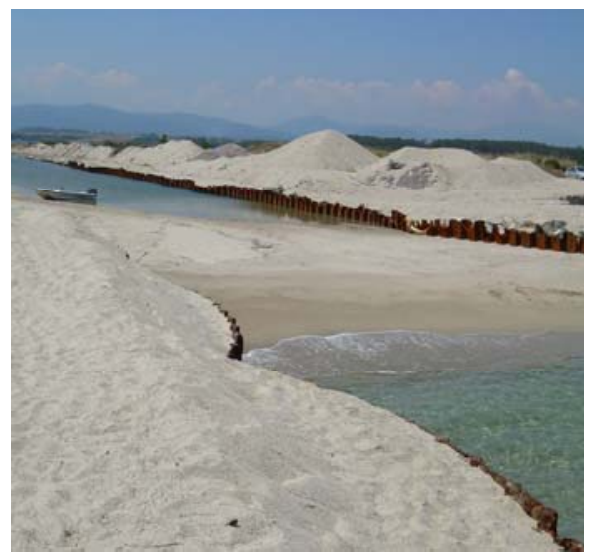

(b)

Figure 1. (a) Configuration actuelle de l'embouchure ; (b) Barre de sable dans le grau.

L'entretien et le réaménagement du grau, ont un impact socio-économique important. Dans l'objectif du maintien des activités professionnelles de l'étang et de la préservation écologique du site, ce travail vise à aboutir à des solutions d’aménagement et de gestion permettant d'établir une communication durable entre l'étang et la mer. 


\section{Résultats}

En complément d'une étude de terrain approfondie, des modélisations numériques ont été réalisées. La chaîne de calcul mise en place est constituée de deux principales étapes réalisées à l'aide des logiciels REFONDE et REFLUX, développés intégralement par le CETMEF, qui modélisent respectivement l'agitation de la houle et les courants. La modélisation des courants a été réalisée en considérant les forçages suivants : vent, marée, courant (des rivières du bassin versant) et houle, via un couplage avec REFONDE (DEBAILLON et al., 2001). Celle-ci a fait l'objet d'une étape conséquente de calibrage du modèle réalisée sur la base d'une large campagne de mesures sur site.

\subsection{Agitation}

Nous nous concentrons ici sur deux conditions d'agitation représentant les conditions de houles les plus fréquentes sur le secteur (de provenance $150-165^{\circ} \mathrm{N}$ et $0-15^{\circ} \mathrm{N}$ ).

L’agitation générée par une houle de secteur sud est supérieure à celle générée par une houle de secteur nord. Ces fortes houles sont susceptibles d'être à l'origine d'apports sédimentaires devant le grau et dans le chenal.

\subsection{Analyse hydrosédimentaire}

Les courants générés dans l'étang sont, de manière générale, très faibles et sensibles aux vents. La condition de climat général générée par une houle de secteur $150-165^{\circ} \mathrm{N}$ engendre des vitesses importantes côté mer et un transit sédimentaire conséquent vers le nord. Le grau fait office d'épi, provoquant un engraissement au sud de l'ouvrage et une érosion au nord de celui-ci, générée par les forts désordres que l’on a pu reproduire en modèle (voir figure 2a). Dans le chenal, les conditions de houles sont telles que les courants sont importants et dirigés vers l'intérieur de l'étang sur la plus grande partie du cycle de marée. La conservation d'un flux entrant important dans le chenal assure un entretien naturel du grau dans de telles conditions.

A partir du moment où le flux est entrant et suffisamment conséquent, des particules sédimentaires sont transportées via le chenal jusque dans l'étang. Dès lors que les vitesses chutent avec un accroissement significatif de la section hydraulique, le sable est susceptible de se déposer. Dans ce cas, les sédiments se déposeront à la sortie du chenal sur le fond de l'étang. Ces sédiments sont d'origine marine mais sont aussi potentiellement issus de l'érosion des berges sableuses du chenal.

Lors de conditions de secteur $0-15^{\circ} \mathrm{N}$ (voir figure $2 \mathrm{~b}$ ), les vitesses dans le chenal sont moindres et ceci peut engendrer un dépôt sédimentaire. Le phénomène est amplifié avec des conditions climatologiques plus sévères. Dans le cas proposé, un vent plus important augmente considérablement les courants au droit de l'ouvrage côté mer, renforçant ainsi la capacité du transport sédimentaire. Les sédiments entrainés par de fortes vitesses dans le chenal s’y déposent rapidement, via la chute brutale des vitesses. Ce phénomène conduirait donc à l'obstruction du grau. 
La connaissance de la Mer :

un vecteur du développement durable en Méditerranée

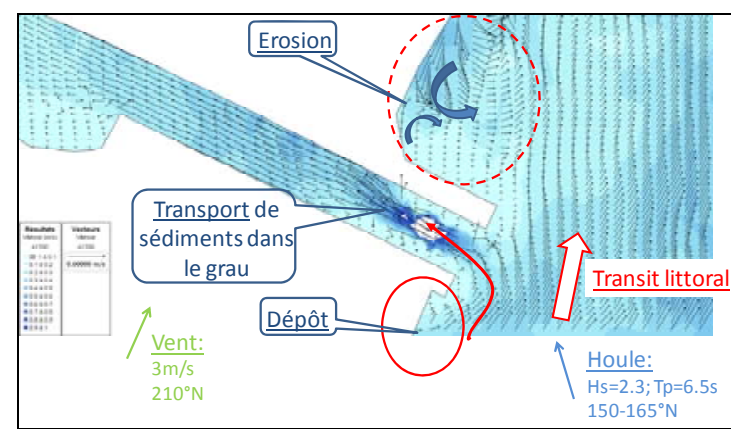

(a)

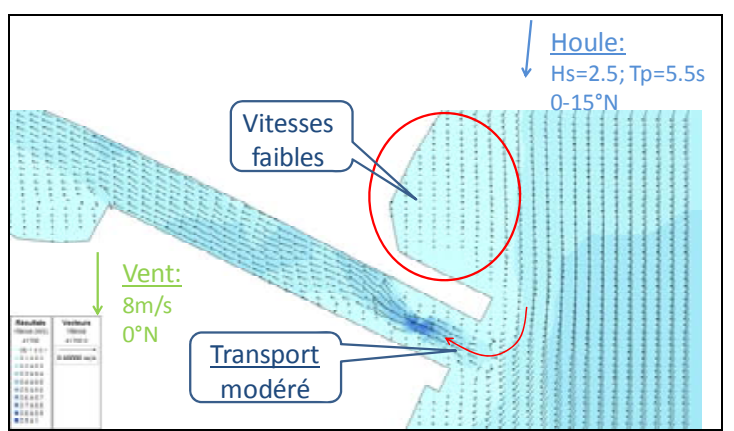

(b)

Figure 2. Modélisations des courants ciblés sur le grau :

(a) Houle de secteur $150-165^{\circ} \mathrm{N}$; (b) Houle de secteur $0-15^{\circ} \mathrm{N}$.

\section{Conclusions}

Les mécanismes induisant l'obstruction du chenal ont été mis en évidence. Les apports sédimentaires marins (générés par les houles de secteur sud), les apports générés par l'érosion des berges du chenal, la faible hauteur d'eau qui limite les effets de chasse dans le chenal et la longueur de celui-ci, qui engendre des problématiques d'entretien, sont les points principaux dont les aménagements doivent tenir compte.

Trois stratégies sont envisagées, les deux premières abandonnant le grau actuel et créant une ouverture, soit en lieu et place du grau historique, soit à l'endroit où le lido est le moins large (pour un entretien plus léger). Il s'agirait alors d'artificialiser des zones naturelles où, côté mer, le trait de côte n'a pas de discontinuité et où le milieu est fortement colonisé de plantes côté étang. La troisième stratégie qui consiste à réaménager l'ouverture actuelle est préférée.

L'objectif d'un auto-entretien naturel à $100 \%$ n'est pas tenable mais la part d'autoentretien peut être optimisée. Quelle que soit la stratégie d'aménagement sélectionnée, il est nécessaire de mettre en œuvre des actions permettant de réactiver les échanges entre l'étang et la mer en augmentant la section hydraulique du chenal (curage) mais aussi de réduire les apports sédimentaires d'origine marine (ouvrage) et issus des berges (fixation des sédiments).

\section{Références bibliographiques}

BRGM (1996). Cartographie des trois grands étangs corses : évolution des rivages des étangs de Chiurlinu (Biguglia), Diana et Urbino de 1948 à 1996, DRIRE Corse, OEC. BRGM (2010). Atlas littoral de la Plaine Orientale, Rapport BRGM/RP-59058-FR DEBAILLON D., SERGENT P., ZHANG B. (2001). Morphological evolution behind a detached shore-parallel breakwater, Coastal Dynamics, Lund (Suède), pp 46-54. MONTIGNY F. (2008). Impact des activités agricoles et anthropiques sur l'étang d'Urbino. Conservatoire de l'Espace Littoral et des Rivages Lacustres, Univ. de Corse 